\title{
DO REWARDLESS ORCHIDS SHOW A POSITIVE RELATIONSHIP BETWEEN PHENOTYPIC DIVERSITY AND REPRODUCTIVE SUCCESS?
}

\author{
Ann Smithson, ${ }^{1,3}$ Nicolas Juillet, ${ }^{2}$ Mark R. Macnair, ${ }^{1}$ and Luc D. B. Gigord ${ }^{2}$ \\ ${ }^{1}$ Department of Biological Sciences, University of Exeter, Hatherly Laboratories, Prince of Wales Road, Exeter EX4 4PS, UK \\ ${ }^{2}$ Department of Ecology and Evolution, Biology Building, University of Lausanne, Lausanne, CH-1015, Switzerland
}

\begin{abstract}
Among rewardless orchids, pollinator sampling behavior has been suggested to drive a positive relationship between population phenotypic variability and absolute reproductive success, and hence population fitness. We tested this hypothesis by constructing experimental arrays using the rewardless orchid Dactylorhiza sambucina, which is dimorphic for corolla color. We found no evidence that polymorphic arrays had higher mean reproductive success than monomorphic arrays for pollinia removal, pollen deposition, or fruit set. For pollinia removal, monomorphic yellow arrays had significantly greater reproductive success, and monomorphic red the least. A tendency for yellow arrays to have higher pollen deposition was also found. We argue that differential population fitness was most likely to reflect differential numbers of pollinators attracted to arrays, through preferential long-distance attraction to arrays with yellow inflorescences. Correlative studies of absolute reproductive success in 52 populations of $D$. sambucina supported our experimental results. To our knowledge this is the first study to suggest that attraction of a greater number of pollinators to rewardless orchids may be of greater functional importance to population fitness, and thus ecology and conservation, than are the behavioral sequences of individual pollinators.
\end{abstract}

Key words: conservation; corolla color; Dactylorhiza sambucina; Orchidaceae; phenotypic diversity; pollination; population fitness.

\section{INTRODUCTION}

Population diversity, both genetic and phenotypic, is considered beneficial to population fitness and species persistence, as it provides the raw material for adaptation to a changing environment (Frankham et al. 2002). Much effort has been invested in quantifying neutral genetic diversity within and between populations, despite the fact that neutral and adaptive genetic diversity are seldom strongly correlated (Reed and Frankham 2001). In some cases phenotypic diversity is expected to be directly and positively correlated with population fitness. For example, the number of alleles controlling self-incompatibility in plants is positively related to population reproductive success and persistence, since allelic diversity increases the number of potential mates (Young et al. 2000). Experiments studying such direct relationships are rare, however.

Rewardless orchids provide a particularly interesting case study for studying direct positive relationships between population phenotypic diversity and population fitness. While most animal-pollinated plants provide nectar or pollen reward to entice pollinators into visitation, rewardless orchids rely on deception of pollinators without mimicry to achieve pollen transfer

Manuscript received 14 September 2005; revised 16 May 2006; accepted 30 May 2006; final version received 27 June 2006. Corresponding Editor: R. Mitchell.

${ }^{3}$ E-mail: A.Smithson@exeter.ac.uk
(Heinrich 1975, Dafni 1984, Ackerman 1986, Nilsson 1992). Rewardlessness is rare generally across all plant families, but more common in orchids (one third of species; van der Pijl and Dodson 1966). Rewardless orchids have notably high within-population phenotypic diversity, especially for corolla color (Heinrich 1975, Nilsson 1980, Dafni 1984, Slater and Calder 1988, Cropper and Calder 1990, Ackerman and Galarza-Pérez 1991), consistent with the hypothesis that phenotypic diversity is positively related to population fitness.

Pollinators' avoidance-learning behavior while sampling rewardless orchids is suggested to drive a positive relationship between the phenotypic variability of a population of a rewardless species and the total number of pollinator visits a population receives, and thus total reproductive success and population fitness (Heinrich 1975, Ferdy et al. 1998). It has been predicted that pollinators take more flower visits to learn to avoid a rewardless species in a population where phenotypic diversity is high, so that the cumulative number of visits received, and reproductive success accrued, should increase with increasing population phenotypic variability (Heinrich 1975, Nilsson 1980, Dafni 1984, Cropper and Calder 1990, Ackerman and Galarza-Pérez 1991). It is therefore expected that the population fitness of a rewardless species should reach a maximum when many floral variants are present at equal frequencies in a population (Ferdy et al. 1998). The hypothesis assumes that reproductive success is strongly pollinator limited, 
which is common in rewardless species (Neiland and Wilcock 1998, Tremblay et al. 2005). Since a positive relationship between diversity and population fitness would result in the highest population seed production in phenotypically diverse populations, testing the hypothesis is important for understanding the persistence and conservation ecology of populations of rewardless species, especially orchids (Ackerman 1986, Ferdy et al. 1998). To date, the only experimental test of this hypothesis, using captive pollinators foraging on painted single flowers, found weak evidence for a positive relationship (Dukas and Real 1993).

Negative frequency-dependent selection is suggested to be the evolutionary force maintaining corolla color polymorphisms within populations of rewardless orchids (Ackerman 1986, Smithson and Macnair 1997, Gigord et al. 2001, Smithson 2001). If a pollinator samples proportionately more inflorescences with rare corolla colors than expected by chance while searching a patch for reward, this drives a negative relationship between color morph frequency and the reproductive component of fitness (Ackerman 1986, Smithson and Macnair 1997, Gigord et al. 2001, Smithson 2001). However, while pollinator behavior may drive an individual fitness advantage for plants with rare colors, this does not necessarily imply that individual pollinators sample more plants in diverse populations, since this depends on the avoidance learning mechanism adopted (Smithson and Macnair 1997). In previous experiments, captive pollinators sampled disassortatively on color morphs of artificial flowers that were rewardless but switched to assortative visitation by color morph on encountering a reward (Smithson and Macnair 1997, Dukas and Real 1993). Such visitation caused negative frequency dependence but resulted in the cumulative number of visits to rewardless color morphs in arrays not being positively correlated with their color diversity (Smithson and Macnair 1997, Dukas and Real 1993).

Are we therefore justified in expecting a positive relationship between phenotypic diversity and population fitness in the field? We carried out the first field experimental test of this hypothesis, using populations of a rewardless plant to quantify population fitness. We estimated the population fitness as the absolute reproductive success of a population summed across all plants in a population, and we focused on a species of rewardless orchid using corolla color as the phenotypic trait of interest. First, we conducted a replicated manipulative field experiment using potted orchids to construct arrays that were either monomorphic or polymorphic for corolla color, while maintaining constant density. Second, we tested for correlations between absolute reproductive success and corolla color diversity in natural populations. The rewardless European orchid Dactylorhiza sambucina is the ideal model study system, as it has a striking red-yellow corolla color dimorphism that is discriminated by pollinators
(Nilsson 1980, Smithson 2001, Gigord et al. 2002, Jersáková et al. 2006). This species has been used previously in manipulative experiments, and most natural populations of the species are polymorphic (mean morph frequency 53\% yellow, $n=174$; Smithson 2001, Gigord et al. 2001).

\section{Materials And Methods}

Dactylorhiza sambucina (see Plate 1) is a locally abundant, European arctic-alpine rewardless orchid that flowers in spring (roughly May) and is pollinated by newly emerged bumble bee queens across the species range. It grows in open grassy meadows, with a preference for neutral to alkaline soils (Nilsson 1980). Reproductive success is strongly pollinator-limited, and plants are self-compatible (Nilsson 1980, Peter and Johnson 2006). Each corm produces one inflorescence per year with a mean $( \pm \mathrm{SE})$ of $12.24 \pm 0.13$ (yellow) and $12.32 \pm 0.13$ (red) flowers per inflorescence. Each flower has two pollinia, which are removed whole either singly or more commonly in pairs (L. D. B. Gigord, M. R. Macnair, and A. Smithson, unpublished manuscript), but pollen is deposited gradually onto stigmas in clumps (massulae). Geitonogamy rates for this species are unknown, although pollinarium bending occurs after removal by a pollinator, which is thought to be an adaptation to reduce geitonogamy (Nilsson 1980). Experiments with dyed pollinia suggest substantial between-morph pollen transfer, although pollinia dyeing reduced pollinia removal (R. Gala and A. Smithson, unpublished data). While flowers open from bottom to top sequentially as plants come into flower, if unpollinated, all flowers on an inflorescence can be open simultaneously for the majority of the flowering period, which can last for up to three weeks depending on weather conditions (Nilsson 1980; A. Smithson, unpublished data).

The manipulative experiment was centered on the Causse Noir, France $\left(44^{\circ} 10^{\prime}\right.$ N 3 $20^{\prime}$ E) during 2004. Populations of D. sambucina occur in the surrounding Cévennes National Park (mean morph frequency $\pm \mathrm{SE}$ $=69 \% \pm 3 \%$ yellow), but they are rare on the Causse. We used a $D$. sambucina population in the periphery region of the National Park as a source, and translocated plants just before flowering to one of 21 array locations on the Causse. Array locations were selected to be of relatively similar habitat to each other and natural populations, with abundant pollinators, protected from disturbance, and at least $1.5 \mathrm{~km}$ apart and $5 \mathrm{~km}$ from any natural population. We haphazardly selected $D$. sambucina from the source population to give an equal number of red and yellow inflorescences, excavated gently with natural soil, and potted individually. We maintained inflorescences in mesh bags to exclude pollinators, which were removed after placing plants into field arrays.

The 21 arrays were randomly allocated to one of three treatments: monomorphic yellow, monomorphic red, 
and 50\% yellow: $50 \%$ red; with seven arrays per treatment. Maps were used to check that there was no chance spatial clustering of arrays to treatments. Each array contained 30 inflorescences, randomly allocated to arrays after controlling to ensure total and variance in flower number were equal across arrays and between colors within mixed arrays (each array contained two five-flowered inflorescences, four six-flowered, six sevenflowered, four eight-flowered, four nine-flowered, four 10-flowered, two 11-flowered, two 12-flowered, and two 13-flowered inflorescences, to total 256 flowers across 30 inflorescences per array; half of each was allocated to each color for mixed arrays). Each array measured $9 \times 9$ $\mathrm{m}$ with $1 \mathrm{~m}$ grid size, giving 81 possible positions, and 30 inflorescences were randomly allocated to available positions. A regular grid was not used, as pollinating bumble bees can follow a trap-line when foraging, which could affect their choice behavior (Smithson 1996), and furthermore a random arrangement more closely approximates the distribution of the species in the field. Inflorescences were placed into position and exposed to pollinators by removing mesh bags on the evening of 15 May, when all experimental plants contained at least five open flowers, and was the peak flowering period for $D$. sambucina. Plants were exposed for nine days, and during this time all flowers had opened and begun to fade. Arrays were watered daily, but a hot period led to loss of one array (monomorphic red) during exposure and to some flowers and inflorescences within other arrays. After exposure, inflorescences were bagged and returned to a central holding area where flower number, pollinia removal and numbers of stigmas receiving pollen were quantified for each inflorescence. Inflorescences were haphazardly arranged in the holding area, watered regularly, and, after three weeks, fruit set was quantified. Each time reproductive success was assessed, counters were blind to treatment, and inflorescences were checked in a haphazard order with respect to treatment. After the experiment, plants were carefully returned to their marked position in the source population.

We tested whether phenotypic diversity and absolute reproductive success were related by comparing pollinia removal, pollen deposition, and fruit set among treatments. The best way to quantify absolute reproductive success would have been to have summed estimates of reproductive success for all plants within each array, considering pollinia removal, pollen deposition, and fruit set separately, and then testing for significant differences among treatments. This approach proved not possible, because some individual flowers and inflorescences were occasionally lost through the experiment, which could distort results if there was a bias in loss rate according to array or corolla color. Therefore we estimated absolute reproductive success per array as the mean reproductive success within each array on a per plant basis. This was calculated for each plant by dividing pollinia removal, pollen deposition, and fruit set by the number of flowers present at the assessment of pollinia removal and thus available to pollinators, which allowed us to control for flower loss. Plants that died were excluded from assessment. This approach is valid, assuming arrays and treatments did not differ significantly in the number of flowers available to pollinators at any time, but we were unable to check reproductive success on a daily basis so could not control for differential timing of flower loss across treatments. We compared mean pollinia removal, pollen deposition, and fruit set per array among treatments using ANOVA after square-root transformation of data to ensure normality and homogeneity of variances. The total numbers of flowers lost, assessed at pollinia removal, and inflorescence loss, quantified when fruit set was assessed, were also compared by ANOVA after squareroot transformation of data, to test for bias according to treatment or inflorescence color. The monomorphic red array lost during the experiment was excluded from data analysis, giving a total sample size of 20 arrays.

The correlative study was carried out in spring 1999, when we studied 52 populations of $D$. sambucina in the center of the species range in France and the northern edge in Scandinavia (L. D. B. Gigord, M. R. Macnair, and A. Smithson, unpublished manuscript). Populations were located in France in the Alps $\left(44.1^{\circ} \mathrm{N}, 6.35^{\circ} \mathrm{E}, N=\right.$ 3 ), in the Massif des Cévennes $\left(44.1^{\circ} \mathrm{N}, 3.3^{\circ} \mathrm{E}, N=15\right)$, in the Pyrénées $\left(42.3^{\circ} \mathrm{N}, 1.5^{\circ} \mathrm{E}, N=4\right)$, in Andorra $\left(42.4^{\circ} \mathrm{N}, 1.3^{\circ} \mathrm{E}, N=5\right)$, in Swedish Upland $\left(59.5^{\circ} \mathrm{N}\right.$, $\left.18.4^{\circ} \mathrm{E}, N=1\right)$, in Gotland $\left(57.4^{\circ} \mathrm{N}, 19.0^{\circ} \mathrm{E}, N=4\right)$, and finally in the Finnish Aland archipelago $\left(60.1^{\circ} \mathrm{N}, 19.5^{\circ}\right.$ E, $N=20$ ). Populations were selected to maximize the range of morph frequencies sampled within each area and to be accessible, although where large areas of $D$. sambucina occurred sample areas were $\geq 5 \mathrm{~km}$ apart. Data for as many populations as possible were collected using two teams within one flowering season. We quantified the frequency of yellow and red inflorescences during flowering by counting. To estimate reproductive success, 20 pairs of inflorescences were selected randomly by choosing the nearest inflorescence at appropriate intervals along a transect through the center of each population, and matching each chosen inflorescence to one of alternate color that had similar floral traits (flower number and inflorescence height), so that an equal number of inflorescences were sampled for each morph. Inflorescences were marked, and after flowers began to wither estimates of reproductive success (pollinia removal, number of flowers receiving pollen depositions, and fruit set) were quantified. Population reproductive success as an index of population fitness was calculated first by finding the mean reproductive success for each inflorescence, by dividing pollinia removal, pollen deposition, and fruit set by the number of flowers available per inflorescence, to control for any variation in plant size between populations. Absolute reproductive success per population was then calculated by finding the mean estimate for each flower color, and 

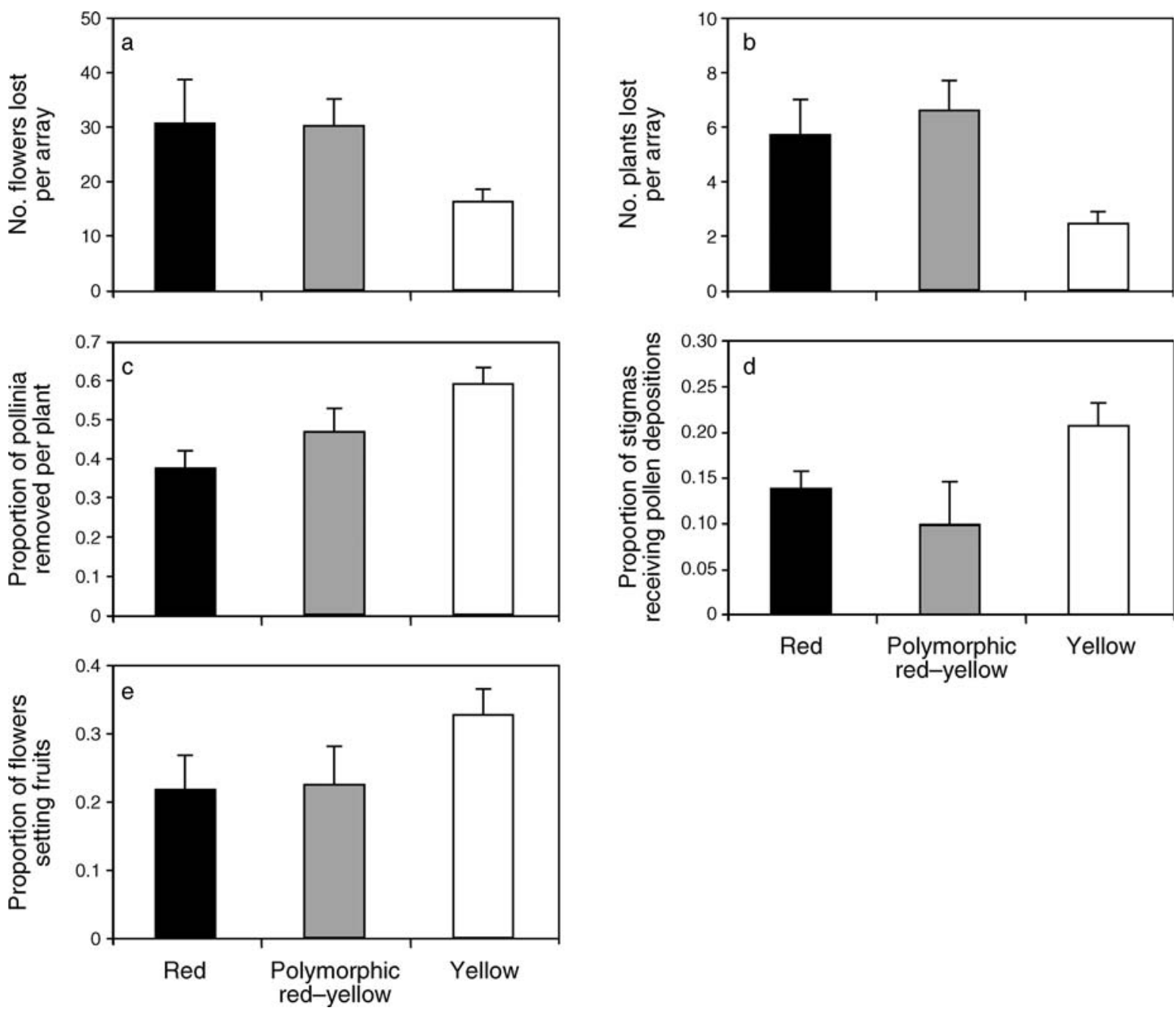

FIG. 1. Reproductive success and flower and inflorescence loss in experimental arrays of Dactylorhiza sambucina with three treatments: monomorphic for red corolla color (solid bars), polymorphic (50\% red, $50 \%$ yellow; shaded bars), or monomorphic for yellow (open bars). (a) Total number of flowers damaged and lost to the experiment during the nine-day exposure period. (b) Number of inflorescences lost per array at the end of the holding period when fruit set was recorded. (c) Proportion of pollinia removed per flower per array. (d) Proportion of stigmas with pollen depositions per flower per array. (e) Proportion of fruits set per flower per plant in each array. Histograms show mean values, error bars represent + SE, and sample sizes were seven (monomorphic yellow and polymorphic arrays) or six (monomorphic red arrays).

then multiplying by its frequency in the population so that the means for each flower color were weighted by morph frequency. This produced two indices of absolute reproductive success, one for each color, which were then summed to produce the index for each population. If there was a relationship between phenotypic diversity and absolute reproductive success, we expected that a regression between morph frequency and the three indices of reproductive success would have a significant quadratic component, indicating a peak in reproductive success at an intermediate frequency. Each variable was arcsine-transformed before analysis.

All statistical analyses were carried out on SPSS for Windows (version 11, SPSS, Chicago, IL, USA). Where means are given, standard errors are also quoted.

\section{RESUlTS}

For the manipulative experiment, we quantified the total number of flowers that had been available per array for pollinators at the end of the exposure period, to test whether this parameter differed significantly between treatments. Arrays receiving different treatments did not differ significantly in the total numbers of flowers lost or damaged during this period (Fig. 1a; ANOVA, $F_{2,17}=2.594, P=0.10$ ). While yellow arrays tended to have more open flowers available for pollinators, this was not statistically significant either for flowers available for pollinia removal (ANOVA, $\left.F_{2,17}=2.85, P=0.09\right)$ or for flowers with stigmas available for pollen deposition (ANOVA, $F_{2,17}=3.02, P$ $=0.08)$. At fruit assessment, however, there was 


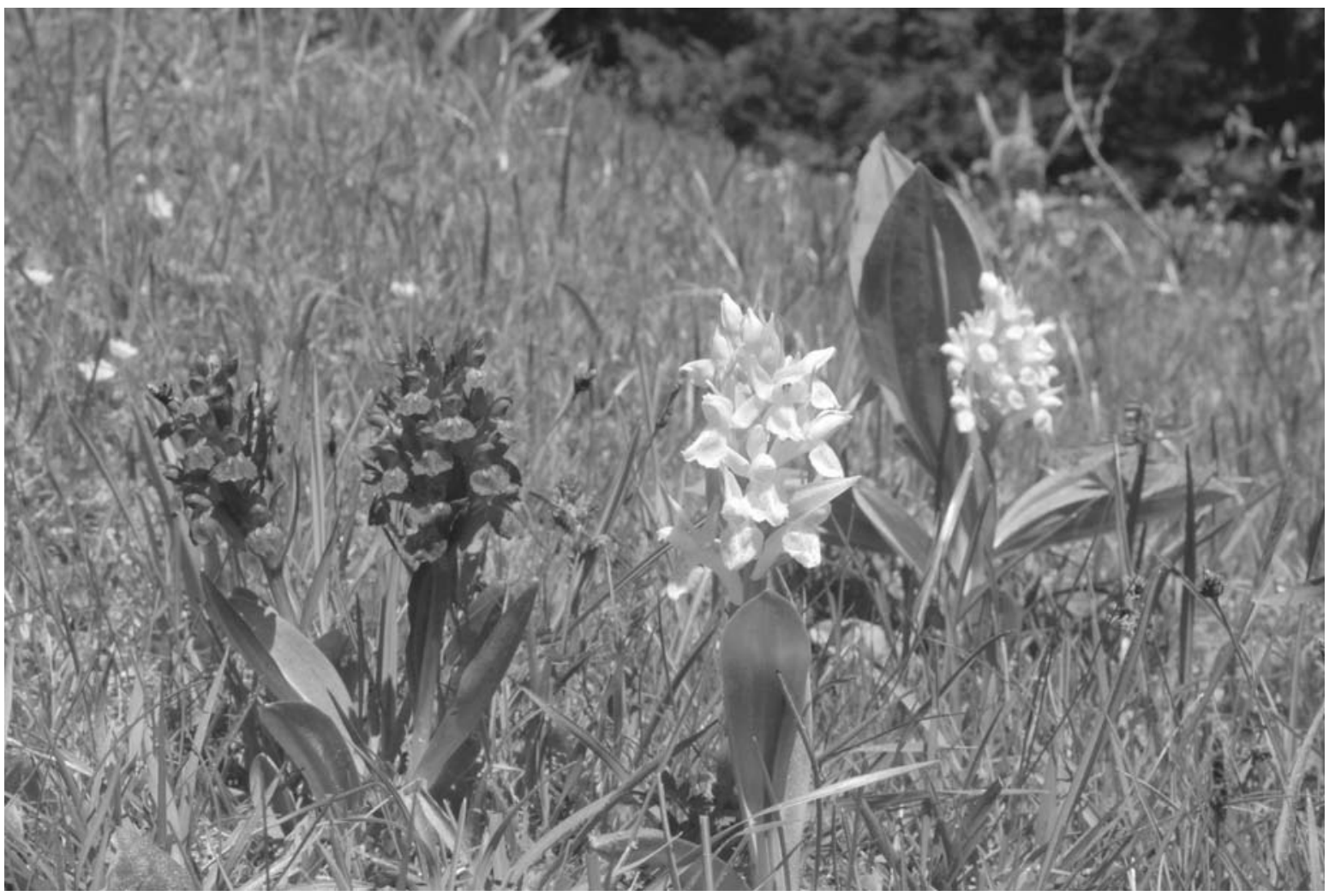

Plate 1. Dactylorhiza sambucina. Photo credit: N. Juillet.

significantly less inflorescence loss from monomorphic yellow arrays than from other treatments (Fig. 1b; ANOVA, $F_{2,17}=5.36, P=0.02$ ), indicating differential loss of inflorescences in the holding area after exposure. On average, $4.85 \pm 0.69$ inflorescences were lost per array here. To correct for any possible effects of differential flower and inflorescence loss through the experiment, total reproductive success was estimated as means per flower or inflorescence (see Materials and Methods). The effect of color morph on the reproductive success of individual plants did not differ between those suffering inflorescence loss while in the holding area setting fruits and those that did not suffer such inflorescence loss, either for pollinia removal $\left(F_{1,575}=\right.$ $0.54, P=0.47)$ or pollen deposition $\left(F_{1,575}=2.80, P=\right.$ 0.10 ).

If phenotypic diversity increased pollinator visitation, we expected that polymorphic arrays should have had significantly higher pollinator-mediated reproductive success than monomorphic arrays. We found that mean pollinia removal in arrays differed significantly between treatments (Fig. 1c; ANOVA, $F_{2,17}=4.77, P=0.02$ ). However, these differences were not in the direction predicted: Monomorphic yellow arrays had the highest pollinia removal, with polymorphic arrays intermediate, and monomorphic red arrays the least (Fig. 1c). There is no significant difference in pollinia removal between monomorphic arrays combined and polymorphic arrays
(ANOVA, $F_{1,18}=0.04, P=0.84$ ). It appeared (Fig. 1c) that pollinia removal increased linearly with the number of yellow inflorescences present (i.e., ordering of removal, lowest to highest: red monomorphic, polymorphic, yellow monomorphic). To test for the significance of this pattern, we carried out a post hoc orthogonal linear contrast through the contrast function in SPSS ANOVA, which proved highly significant (ANOVA, $F_{1,17}=9.52, P=0.007$ ).

Mean pollen deposition and mean fruit set showed similar patterns to those for pollinia removal, in that yellow monomorphic arrays tended to have the highest reproductive success (Fig. 1d, e). The trend for higher pollen deposition onto yellow arrays was only marginally significant (ANOVA, $F_{2,17}=3.59, P=0.05$ ), while there were no significant differences between treatments for fruit set (ANOVA, $F_{2,17}=1.59, P=0.23$ ). As for pollinia removal, polymorphic arrays did not have significantly greater reproductive success either for pollen deposition (ANOVA, $F_{1,18}=3.82, P=0.07$ ) or fruit set (ANOVA, $F_{1,18}=0.44, P=0.52$ ), and for pollinia removal the trend was toward least pollen deposition onto polymorphic arrays. Post hoc orthogonal linear contrasts with treatments ordered as for pollinia removal were not significant (pollen deposition, ANOVA, $F_{1,17}=3.27, P=0.09$; fruit set, ANOVA, $F_{1,17}$ $=2.58, P=0.13$ ). 
Comparing reproductive success for inflorescences within polymorphic arrays only, yellow inflorescences consistently had the highest relative reproductive success, which was significant for pollinia removal (paired $t$ test, $t_{6}=4.07, P=0.01$ ) and fruit set (paired $t$ test, $t_{6}=$ 2.48, $P=0.05$ ), but not for pollen deposition (paired $t$ test, $\left.t_{6}=1.92, P=0.10\right)$. The relative fitness advantage for yellow inflorescences within mixed arrays was small however: 1.13, 1.10, and 1.12 for pollinia removal, pollinia deposition, and fruit set, respectively, where relative fitness is estimated as the ratio of reproductive success of yellow inflorescences to half the reproductive success of yellow plus red.

For the correlative study, we expected a peak in population fitness at an intermediate color morph frequency of $D$. sambucina corresponding to maximal color diversity. However a quadratic regression of morph frequency on the three indices of absolute reproductive success did not significantly increase the proportion of the variance explained over linear regression (Fig. 2a-c, Table 1; significance of difference between linear and quadratic regression, pollinia removal, $F_{2,49}=1.00, P=0.38$; pollen deposition, $F_{2,49}=0.96$, $P=0.39$; fruit set, $F_{2,49}=0.98, P=0.38$ ). Furthermore, in quadratic regression none of the quadratic coefficients were significant for the three indices of reproductive success, while all linear coefficients were significant (Table 1). All three indices of absolute reproductive success and thus population fitness increased linearly with the proportion of yellow flowers present in the population, and thus do not match the expectation for an intermediate peak (Table 1, Fig. 2a-c).

\section{Discussion}

Contrary to the prediction that phenotypically diverse populations of rewardless orchids should have maximal population fitness (Heinrich 1975, Ferdy et al. 1998), polymorphic arrays of $D$. sambucina did not have higher mean reproductive success than monomorphic arrays, either for pollinia removal, pollen deposition, or fruit set. This result was supported by correlative studies in natural populations, where absolute reproductive success did not increase at maximal phenotypic diversity. The results of our experiments did not support the alternative hypothesis that population fitness should be independent of the frequency of color morphs in populations (Smithson and Macnair 1997). Instead we found, in array experiments, that pollinia removal increased from red to polymorphic to yellow arrays, and pollen deposition and fruit set follow a similar pattern. In the correlative study, we found that all measures of absolute reproductive success were positively correlated with the frequency of yellow $D$. sambucina. Our correlative results could be biased, because morph frequencies are variable, spatially substructured, and populations in Scandinavia tend to have more variable and red-biased morph frequencies and be more pollinator-limited than French populations
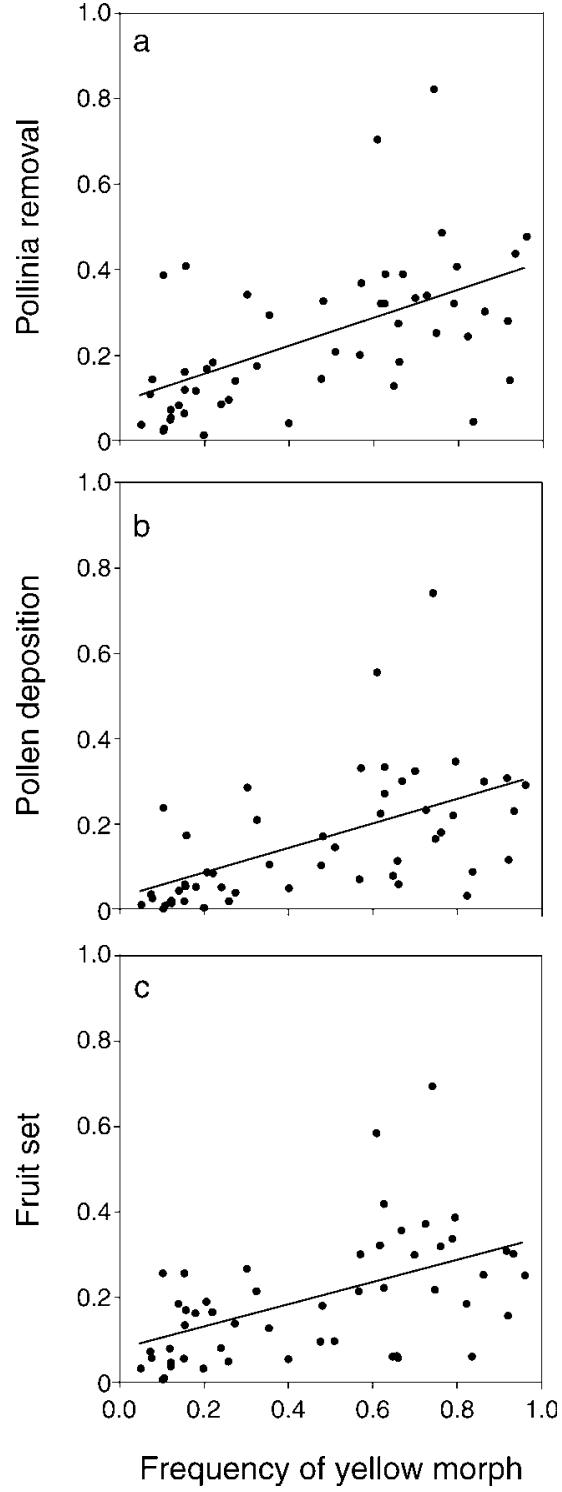

FIG. 2. The relationship between absolute reproductive success, expressed as a proportion of the number of flowers available per plant averaged across plants sampled, and the frequency of the yellow morphs in natural populations, showing three indices of absolute reproductive success from 52 natural populations of D. sambucina. Graph units represent mean proportions per plant of (a) pollinia removed, (b) pollen deposited, or (c) stigmas setting fruits.

(L. D. B. Gigord, M. R. Macnair, and A. Smithson, unpublished manuscript). However, absolute reproductive success and yellow morph frequency remain positively correlated in Scandinavia alone (pollinia removal, $F_{1,23}=4.66, P=0.04, r=0.41$ ), indicating that the availability of yellow inflorescences is the most likely causal variable. Thus our experiments suggested that differences in population fitness for D. sambucina are unlikely to be caused by color diversity per se, but to differences in the abundance of yellow inflorescences. Levin and Kerster (1970) similarly found a positive 
TABLE 1. The relationship between three indices of reproductive success and morph frequency for the correlative study conducted in 1999, as quantified by linear and quadratic regression.

\begin{tabular}{|c|c|c|c|c|c|c|c|c|c|c|}
\hline \multirow[b]{3}{*}{ Variable } & \multirow{2}{*}{\multicolumn{3}{|c|}{ Linear regression }} & \multirow{2}{*}{\multicolumn{3}{|c|}{ Quadratic regression }} & \multicolumn{4}{|c|}{ Regression coefficients } \\
\hline & & & & & & & \multicolumn{2}{|c|}{ Linear regression } & \multicolumn{2}{|c|}{ Quadratic regression } \\
\hline & $r^{2}$ & $F_{1,50}$ & $P$ & $r^{2}$ & $F_{2,49}$ & $P$ & Coefficient & $P$ & Coefficient & $P$ \\
\hline Pollinia removal & 0.33 & 24.56 & $<0.001$ & 0.35 & 13.48 & $<0.001$ & $0.94 \pm 0.41$ & 0.03 & $-0.37 \pm 0.27$ & 0.17 \\
\hline Pollen deposition & 0.39 & 31.91 & $<0.001$ & 0.43 & 18.21 & $<0.001$ & $1.07 \pm 0.39$ & 0.008 & $-0.44 \pm 0.25$ & 0.08 \\
\hline Fruit set & 0.30 & 21.12 & $<0.001$ & 0.32 & 11.79 & $<0.001$ & $0.84 \pm 0.38$ & 0.03 & $-0.35 \pm 0.24$ & 0.16 \\
\hline
\end{tabular}

Note: Significances of $F$ and coefficients are also indicated.

correlation between frequency of corolla color variants and population fitness in Phlox drumondii through increased attractiveness to pollinators.

What has caused these patterns, and why do they differ from those predicted? Since D. sambucina has reproductive success that is highly pollinator-limited (Nilsson 1980), differential population reproductive success is probably caused by differential pollinator visitation. This could either be through the recruitment of a different number of pollinators to each population, or because the sequences of flowers visited by each individual pollinator is longer in some populations than others, or through a combination of these two patterns. The hypotheses of Heinrich (1975), Smithson and Macnair (1997), and Ferdy et al. (1998) were based on experiments or models exploring the responses of individual pollinators to corolla color through variation in the length of their visitation sequences, and assumed that differences in population fitness would result from such differential sequence lengths. Thus the most parsimonious explanation for our results is that differential population reproductive success predominantly reflected recruitment of a different number of pollinators, such that variance in length of pollinator visitation sequences had a minor effect on population fitness. Differential attraction and recruitment of pollinators to arrays with yellow inflorescences could be either be caused by innate bias toward yellow as perceived by the eyes of bumble bees, which are sensitive to different wavelengths of light compared to human eyes (Peitsch et al. 1992), or because yellow $D$. sambucina appeared particularly conspicuous to bumble bees against the background of vegetation (Spaethe et al. 2001). We have observed in previous experiments that individual pollinators of $D$. sambucina showed a small but significant preference for yellow inflorescences over red, which supports this suggestion (Gigord et al. 2001, 2002, Smithson 2001). Thus yellow D. sambucina may be exploiting a pollinator sensory bias towards yellow, which may increase long-distance attraction and population-level pollinator recruitment. This hypothesis is testable, using a series of pollinator mark-recapture experiments within populations, although this would present a practical challenge.

There are alternative hypotheses that could explain our results. Arrays could have attracted similar numbers of pollinators, but each pollinator could have visited more yellow inflorescences within each array. This interpretation seems less likely as it does not agree with previous experiments, which indicated (1) that foraging bumble bees sampled in a negatively frequency-dependent manner on rewardless artificial flowers (Smithson and Macnair 1997), and (2) a fitness advantage for rare morphs within arrays of D. sambucina (Gigord et al. 2001). Furthermore, it would be difficult to explain the maintenance of red inflorescences within these populations if there is consistent pollinator avoidance of red inflorescences. Our results may be biased, as there may have been differential loss of pollinated vs. nonpollinated flowers according to inflorescence color during our experiment, but this hypothesis was not supported by an analysis of inflorescences lost between assessment at pollinia removal and at fruit set; and, since desiccation was the only cause of inflorescence loss we could identify, this hypothesis seems unlikely. Daily assessments of reproductive success of all inflorescences in each array would have allowed us to quantify flower loss according to pollination status, but this proved beyond the scope of the current study. Furthermore, it is more likely that inflorescences that had set no fruits were misclassified as dead if the inflorescence stem had begun to wither, which would reduce rather than exaggerate any bias. A further alternative hypothesis is that pollinators may have selected on scent cues that could differ between morphs and use these to preferentially locate arrays with many yellow inflorescences. Previous experiments studying the potential for mimicry in $D$. sambucina did not suggest such scent-based selection, however (Gigord et al. 2002).

The significantly higher loss rate for red inflorescences while these were producing fruits suggests that yellow $D$. sambucina could be slightly less susceptible to desiccation than red, at least at this point in the life cycle, although since our plants were potted further experiments are required to test this hypothesis in the natural environment. Correlative studies also suggest that the frequency of the red morph tends to increase with altitude and latitude, as would be expected if colors differ slightly in response to the microenvironment (L. D. B. Gigord, M. R. Macnair, and A. Smithson, unpublished manuscript). The effects of both pollinators and the microenvironment acting simultaneously on 
corolla color have been found by other authors (e.g., Cirsium vulgare; Mogford 1978). Jersáková et al. (2006) suggested that seeds with yellow female parents had a germination rate disadvantage, which suggested other environmental selection pressures may act differentially on the morphs of D. sambucina, although in orchids laboratory germination does not necessarily reflect that in the field (Smithson 2006). Selection mediated by the microenvironment could thus modify pollinator-induced selection in D. sambucina populations, with the potential to modify population morph frequencies.

In these experiments, we suggest that differential population fitness was likely to be caused by preferential attraction of pollinators into populations with more yellow flowers, while our previous experiments focusing on the causes of maintenance of corolla color diversity in $D$. sambucina have focused on the factors responsible for a relative fitness advantage for rare colors. Can these studies be reconciled? Pollinators may be differentially attracted into populations by long-distance signals from $D$. sambucina, predominantly caused by differences in abundance of yellow inflorescences, but subsequently the sequence of inflorescences sampled by each attracted pollinator within each population may still include both yellow and red inflorescences (Nilsson 1980), may have negative frequency-dependence, and may therefore result in a relative fitness advantage for the rarest color within populations (Smithson and Macnair 1997, Gigord et al. 2001, Smithson 2001). Previous experiments found that relative reproductive success slightly favored yellow morphs in arrays with 50\% yellow morphs (Gigord et al. 2001), which is consistent with the slight relative fitness advantage for yellow found here in polymorphic arrays. We thus suggest overall that pollinator-mediated fitness at the population level is influenced most strongly by differences in the number of individual pollinators attracted into arrays, while within populations relative fitness may be mediated by differences in the visitation sequences of individual pollinators. To our knowledge, this is the first study to suggest that long-distance attraction of greater numbers of pollinators to rewardless species is likely to be of greater functional importance to population fitness, and thus population dynamics and conservation ecology, than behavioral sequences of individual pollinators. Note that population-level selection is unlikely to drive the evolutionary dynamics of corolla color, since the conditions under which such group selection could act as a strong evolutionary force, in comparison to individual selection, seem to be limited (Wade 1985). The behavioral preferences of individual pollinators within populations of rewardless species may thus determine relative morph fitness and evolutionary trajectories, while the total overall number of pollinators attracted to arrays may modify ecological trajectories.

Most color variation within rewardless orchids is continuous rather than discrete (Heinrich 1975, Nilsson 1980, Dafni 1984, Slater and Calder 1988, Cropper and
Calder 1990, Ackerman and Galarza-Pérez 1991). We selected a species with a discrete polymorphism to study here, as it is experimentally tractable. Predators' responses to variation in discrete vs. continuous prey are similar, and theoretically selection on continuous characters should produce the same selective response as that on discrete characters (Clarke 1979, Mani et al. 1990). Thus we expect pollinators to show similar responses to continuous color variation on rewardless species as those described here for D. sambucina, although this deserves further investigation.

Our experimental results predict that, for conservation purposes, no increase in population reproductive success or seed production is expected in rewardless orchid populations if phenotypic diversity is increased. This does not suggest, therefore, that population persistence will necessarily be increased, or extinction likelihood decreased, if phenotypic diversity is high in a population, although there are few studies relating seed production rates to population dynamics in the Orchidaceae. However, the presence of highly varying morph frequencies in populations of $D$. sambucina through Europe (Nilsson 1980, Jersáková et al. 2006; L. D. B. Gigord, M. R. Macnair, and A. Smithson, unpublished manuscript) may reflect genetic drift, or could reflect adaptation to the local environment or pollinator community. Thus ecological advantages to color diversity through permitting adaptation cannot be ruled out, and this hypothesis is testable. Recent research surprisingly suggests that rewardless and rewarding orchids do not differ in terms either of rarity or likelihood of extinction in the Netherlands (Jacquemyn et al. 2004), even though rewardless orchids have far lower fruit set (Neiland and Wilcock 1998, Tremblay et al. 2005). Much further research is required to find predictive strategies for orchid conservation.

\section{ACKNOWLEDGMENTS}

We thank the Parc National des Cévennes for permission and all landowners on the Causse Noir for allowing access, in particular Madame Védrines (Camprieu) and the Passet family (Dargilan). We also thank the Baraille family, Mélanie Glaettli, Mailyn Adriana Gonzalez, Antonina Internicola, Aline Pasche, and Tiziana Ulian for assistance. A. Smithson was funded by a British Ecological Society Small Ecological Project Grant 2246 on the conservation of phenotypic diversity in D. sambucina. N. Juillet and L. D. B. Gigord were funded by Swiss National Science Foundation grant 3100A0-100754/1. The correlative studies were funded by Natural Environment Research Council grant GR3/12106 to A. Smithson and M. R. Macnair, and were carried out while A. Smithson was supported by Natural Environment Research Council fellowship GT5/98/12/TS.

\section{Literature Cited}

Ackerman, J. D. 1986. Mechanisms and evolution of fooddeceptive pollination systems in orchids. Lindleyana 1:108113.

Ackerman, J. D., and M. Galarza-Pérez. 1991. Patterns and maintenance of extraordinary variation in the Caribbean orchid, Tolumina (Oncidium) variegata. Systematic Botany 16:182-194. 
Clarke, B. C. 1979. Evolution of genetic diversity. Proceedings of the Royal Society of London Series B 205:453-474.

Cropper, S. C., and D. M. Calder. 1990. The floral biology of Thelymitra epipactoides (Orchidaceae), and the implications of pollination by deceit on the survival of this rare orchid. Plant Systematics and Evolution 170:11-27.

Dafni, A. 1984. Mimicry and deception in pollination. Annual Review of Ecology and Evolution 15:259-278.

Dukas, R., and L. A. Real. 1993. Learning constraints and floral choice behaviour in bumble bees. Animal Behaviour 46:637-644.

Ferdy, J. B., P. H. Gouyon, J. Moret, and B. Godelle. 1998. Pollinator behavior and deceptive pollination: Learning process and floral evolution. American Naturalist 152:696705.

Frankham, R., J. D. Ballou, and D. A. Briscoe. 2002. Introduction to conservation genetics. Cambridge University Press, Cambridge, UK.

Gigord, L. D. B., M. R. Macnair, and A. Smithson. 2001. Negative frequency-dependent selection maintains a dramatic flower color polymorphism in the rewardless orchid Dactylorhiza sambucina. Proceedings of the National Academy of Sciences (USA) 98:6253-6255.

Gigord, L. D. B., M. R. Macnair, M. Stritesky, and A. Smithson. 2002. The potential for floral mimicry in rewardless orchids: an experimental study. Proceedings of the Royal Society of London B 269:1389-1395.

Heinrich, B. 1975. Bee flowers: a hypothesis on flower variety and blooming times. Evolution 29:325-334.

Jacquemyn, H., R. Brys, M. Hermy, and J. H. Willems. 2004. Does nectar reward affect rarity and extinction probabilities of orchid species? An assessment using historical records from Belgium and the Netherlands. Biological Conservation 121:257-263.

Jersáková, J., P. Kindlmann, and S. S. Renner. 2006. Is the corolla colour dimorphism in Dactylorhiza sambucina maintained by differential seed viability instead of frequencydependent selection? Folia Geobotanica 41:61-76.

Levin, D. A., and H. W. Kerster. 1970. Phenotypic dimorphism and populational fitness in Phlox. Evolution 24:128-134.

Mani, G. S., B. C. Clarke, and P. R. Shelton. 1990. A model of quantitative traits under frequency-dependent balancing selection. Proceedings of the Royal Society of London Series B 240:15-28.

Mogford, D. J. 1978. Pollination and flower color polymorphism, with special reference to Cirsium palustre. Pages 191199 in A. J. Richards, editor. The pollination of flowers by insects. Academic Press, London, UK.

Neiland, M. R., and C. C. Wilcock. 1998. Fruit set, nectar reward, and rarity in the Orchidaceae. American Journal of Botany 85:1657-1671.
Nilsson, L. A. 1980. The pollination ecology of Dactylorhiza sambucina (Orchidaceae). Botaniska Notiser 133:367-385.

Nilsson, L. A. 1992. Orchid pollination biology. Trends in Ecology and Evolution 7:255-259.

Peitsch, D., A. Fietz, H. Hertel, J. de Souza, D. F. Ventural, and R. Menzel. 1992. The spectral input systems of hymenopteran insects and their receptor-based color vision. Journal of Comparative Physiology A 170:23-40.

Peter, C. I., and S. D. Johnson. 2006. Doing the twist: a test of Darwin's cross-pollination hypothesis for pollinarium reconfiguration. Biology Letters 2:65-68.

Reed, D. H., and R. Frankham. 2001. How closely correlated are molecular and quantitative measures of genetic variation? A meta-analysis. Evolution 55:1095-1103.

Slater, A. T., and D. M. Calder. 1988. The pollination biology of Dendrobium speciosum Smith: a case of false advertising? Australian Journal of Botany 36:145-158.

Smithson, A. 1996. Frequency-dependent selection on floral variants by bumblebees Bombus terrestris L. Dissertation (unpublished). University of Exeter, UK.

Smithson, A. 2001. Frequency-dependent selection by pollinators. Pages 237-257 in L. Chittka and J. D. Thomson, editors. Cognitive ecology of pollination: animal behaviour and evolution. Cambridge University Press, Cambridge, UK.

Smithson, A. 2006. Pollinator limitation and inbreeding depression in orchid species with and without nectar rewards. New Phytologist 169:419-430.

Smithson, A., and M. R. Macnair. 1997. Negative frequencydependent selection by pollinators on artificial flowers without rewards. Evolution 51:715-723.

Spaethe, D., J. Tautz, and L. Chittka. 2001. Visual constraints in foraging bumblebees: Flower size and color affect search time and flight behavior. Proceedings of the National Academy of Sciences (USA) 98:3898-3903.

Tremblay, R. L., J. D. Ackerman, J. K. Zimmerman, and R. Calvo. 2005. Variation in sexual reproduction in orchids and its evolutionary consequences: A spasmodic journey to diversification. The Biological Journal of the Linnean Society $84: 1-54$.

van der Pijl, L., and C. H. Dodson. 1966. Orchid flowers: their pollination and evolution. University of Miami Press, Coral Gables, Florida, USA.

Wade, M. J. 1985. Soft selection, hard selection, kin selection and group selection. American Naturalist 125:61-73.

Young, A. G., A. H. D. Brown, B. G. Murray, P. H. Thrall, and C. H. Miller. 2000. Genetic erosion, restricted mating and reduced viability in fragmented populations of the endangered grassland herb Rutidosis leptorrhynchoides. Pages 335-359 in A. G. Young and G. M. Clarke, editors. Genetics, demography and viability of fragmented populations. Cambridge University Press, Cambridge, UK. 